\title{
COBib-Públicas: observatorio de las bibliotecas públicas en España durante la pandemia por Covid-19 basado en noticias digitales
}

COBib-Públicas: an observatory of public libraries in
Spain during the Covid-19 pandemic based on
digital news

\section{Pedro Lázaro-Rodríguez}

Lázaro-Rodríguez, Pedro (2020). "COBib-Públicas: observatorio de las bibliotecas públicas en España durante la pandemia por Covid-19 basado en noticias digitales". Anuario ThinkEPI, v. 14, e13b04.

https://doi.org/10.3145/thinkepi.2020.e14b04

Publicado en IweTe/ el 18 de junio de 2020

\section{Pedro Lázaro-Rodríguez}

https://orcid.org/0000-0002-8756-0507

Universidad de Granada

Departamento de Información y Comunicación

Campus Universitario de Cartuja

Edificio Máximo. 18071 Granada, España

pedrolr@ugr.es

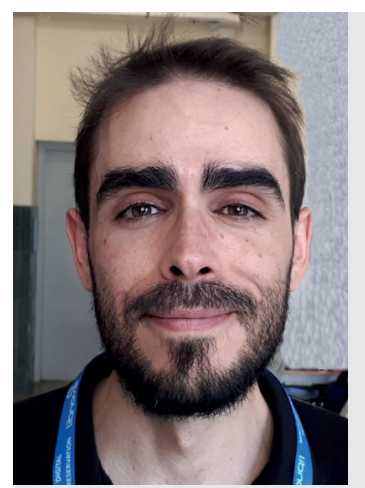

Resumen: En este trabajo se presenta el proyecto COBib-Públicas: observatorio de las bibliotecas públicas en España durante la pandemia por Covid-19 basado en noticias digitales. Se ha implementado en forma de sitio web y aplicación digital incluyendo noticias digitales sobre bibliotecas públicas desde inicios de enero hasta finales de mayo de 2020. El proyecto se concibe como un punto al que acudir para obtener información sobre las bibliotecas públicas españolas durante la pandemia por Covid-19. Se ofrece una mirada general desde el observatorio con un análisis de la evolución del volumen de noticias y los medios digitales que las publican. También, se añade una mirada personal sobre el contenido según los períodos de tiempo considerados, resaltando el protagonismo de la dimensión de lo digital durante el confinamiento, subrayando también la idea de las bibliotecas como vacuna y algunos proyectos de construcción de nuevas bibliotecas. COBib-Públicas puede resultar de interés para las personas dedicadas a la investigación en biblioteconomía y documentación, a las profesionales del sector e igualmente a la población en general.

Palabras clave: Bibliotecas públicas; España; Observatorio; Pandemias; Covid-19; 2019-nCoV; Medios de comunicación; Medios digitales; Noticias digitales.

Abstract: This work presents the project COBib-Públicas, an observatory of public libraries in Spain during the Covid-19 pandemic based on digital news. The project has been published as a web page including digital news on public libraries from January to May 2020. COBib-Públicas is conceived as a place to get information about Spanish public libraries during the Covid-19 pandemic. It includes a general point of view analyzing the evolution of news and the digital media where it was published. Also, some thoughts from a personal point of view are presented, highlighting three aspects: the relevance of the digital dimension of libraries during the confinement, the idea of libraries as a vaccine, and some news on projects to build new libraries. COBib-Públicas may be of interest to library science researchers, librarians, as well as the general public. 
Keywords: Public libraries; Spain; Observatory; Covid-19; 2019-nCoV; Pandemics; Media; Digital journalism; Digital news.

\section{Nota previa}

Dado que en este trabajo se presenta un proyecto implementado en forma de sitio web, se facilita antes que nada su url:

https://wpd.ugr.es/ pedrolr/cobib-publicas/

\section{Introducción}

En este trabajo se presenta la web COBib-Públicas: observatorio de las bibliotecas públicas en España durante la pandemia por Covid-19 basado en noticias digitales. El nombre abreviado del observatorio es COBib-Públicas, forma que recoge la alusión a la Covid-19 (CO), al observatorio (OB), y a las bibliotecas públicas (Bib-Públicas). COBib-Públicas se ha implementado en la web como aplicación digital abierta, recogiendo en un único punto las noticias sobre bibliotecas públicas en medios digitales de España desde inicios de enero hasta finales de mayo de 2020. Dado el carácter dinámico de la web y su función a modo de observatorio, se podrá ir actualizando en el futuro.

En el ámbito científico español se han publicado diversos trabajos sobre medios de comunicación en relación con la crisis sanitaria generada en torno a la enfermedad Covid-19 y el coronavirus 2019-nCoV. Algunos estudios se han centrado en la desinformación y los bulos (Salaverría et al., 2020), y específicamente en la detección de las fake news (Pérez-Dasilva; Meso-Ayerdi; Mendiguren-Galdospín, 2020). En un sentido similar, se dispone de estudios centrados en la desinformación, en el sesgo ideológico y el sensacionalismo de la cobertura mediática (Masip et al., 2020), o analizando las imágenes falsas del virus difundidas al inicio de la pandemia (Andreu-Sánchez; Martín-Pascual, 2020). Otros trabajos se han centrado en el impacto del coronavirus en el sistema de medios (Casero-Ripollés, 2020), o en la comunicación que se ha ido haciendo al respecto comparándola con crisis sanitarias anteriores (Costa-Sánchez; López-García, 2020).

Una conclusión que puede extraerse de los estudios anteriores es el importante papel y la relevancia de los medios de comunicación durante la crisis sanitaria referida. Desde ese papel y relevancia, y relacionando los medios de comunicación y las noticias digitales con las bibliotecas públicas, surge la idea de COBib-Públicas a modo de observatorio y como punto al que acudir para obtener información sobre bibliotecas públicas en España durante la pandemia por Covid-19 a partir de noticias digitales.

Angulo-Marcial (2009) plantea que el concepto de observatorio carece de una claridad precisa en lo referido a su significado. El autor lleva a cabo una revisión del término a través de sus alcances y aplicaciones analizando la definición ofrecida en múltiples trabajos. Por ejemplo, se acepta de una manera general que los observatorios están destinados a identificar y evaluar la información que se produce sobre un tema, todo con base en la evidencia de la realidad. Pero también se subraya la posibilidad de la perspectiva subjetiva de los observatorios, planteando que están relacionados con lo que se ve que existe desde un lugar, pero sin ser el único y definitivo, tratándose todo más de una conjunción de perspectivas diferentes. Con todo, una conclusión del trabajo de Angulo-Marcial (2009) es que los observatorios son espacios abiertos a la investigación, la creatividad y la innovación. En ese carácter de espacio abierto, desde el que observar y disponer de otras observaciones, es en el que se basa la aplicación COBib-Públicas creada. Todo ello implementado de forma creativa e innovadora con el sitio web planteado.

Como observatorio, COBib-Públicas incluye las noticias sobre bibliotecas públicas en medios digitales de España clasificadas por períodos desde el 9 de enero hasta el 24 de mayo de 2020. Para cada noticia se incluye su título con hiperenlace a su url, el medio digital y la fecha en que fue publicada. Las noticias en cada período se muestran ordenadas por defecto según su fecha, de tal forma que se puede hacer una lectura basada en la evolución de noticias durante la pandemia. Con todo, el objetivo de COBib-Públicas es servir de lugar al que acudir para observar qué se ha ido publicando sobre bibliotecas públicas durante la crisis sanitaria por Covid-19. A su vez, ofrece la posibilidad de observar el tema planteado y una mirada general y personal a su contenido.

"El objetivo de COBib-Públicas es servir de lugar al que acudir para observar qué se ha ido publicando sobre bibliotecas públicas durante la crisis sanitaria por Covid-19" 
La siguiente sección está dedicada a los materiales utilizados para recuperar las noticias digitales en que se basa el observatorio. A continuación, se expone la estructura del sitio web creado, explicando el contenido de sus categorías. Tras ello, se incluye una sección que consiste en una mirada general a las noticias recuperadas por períodos y sobre los medios con más noticias publicadas. Muy unido a ello, se ofrece también una mirada personal de las noticias. Por último, se incluyen unas consideraciones finales para cerrar el trabajo.

\section{Materiales}

Para la recuperación de las noticias que conforman la base de COBib-Públicas se utilizó la base de datos MyNews'. En cuanto a la ecuación de búsqueda, se planteó una que incluyese diversas formas de referirse a las bibliotecas públicas en las diferentes lenguas de España. Para ello, se incluyó también el concepto de "biblioteca municipal". Además, se optó por recuperar las noticias que incluyesen dichos conceptos en el título o subtítulo de las noticias digitales. Para todo ello fue muy útil la búsqueda planteada por Lázaro-Rodríguez, López-Gijón y Herrera-Viedma (2018). También, se tuvo en cuenta que la base de datos no admite truncamientos dentro de expresiones entrecomilladas. La ecuación utilizada quedó de la siguiente forma:

("biblioteca publica" OR "biblioteque publique" OR "bibliotecas publicas" OR "liburutegi publikoa" OR "biblioteques publiques" OR "liburutegi publikoak" OR "biblioteca municipal" OR "bibliotecas municipales" OR "biblioteques municipals" OR "bibliotecas municipais" OR "udal liburutegiak" OR "udal liburutegia") \&xoptions=contentfields=title:subtitle

En cuanto a la ventana temporal, se delimitó para recuperar noticias desde el 9 de enero hasta el 24 de mayo de 2020. Al respecto, cabe explicar que se eligió el 9 de enero de 2020 como fecha de inicio debido a los resultados alcanzados en el trabajo de Lázaro-Rodríguez; Herrera-Viedma (2020). En dicho trabajo los autores analizan el volumen de noticias sobre Covid-19 en medios españoles y detectan que las primeras noticias sobre el tema aparecen en torno al 9 de enero. Por su lado, la fecha de cierre de la ventana temporal se basa en que este trabajo se empezó a elaborar el 25 de mayo de 2020, fecha en que se llevó a cabo también la recuperación de las noticias.

Por último, para la búsqueda se utilizaron los filtros de tipo de medio para medios digitales, y de cobertura geográfica para local, regional y nacional. El motivo de ello es delimitar las noticias al ámbito de España y por el carácter digital de la herramienta COBib-Públicas. Cabe especificar que se ha dispuesto un proyecto en Open Science Framework (OSF) con el mismo nombre que el actual trabajo². En dicho proyecto se encuentran disponibles documentos a los que se hace referencia en este trabajo y en el sitio web. También, en ambos lugares se incluirán en el futuro posibles nuevos documentos. Al respecto, los datos recuperados sobre las noticias digitales se han dispuesto en un archivo csv por columnas para sus títulos, urls, medios de publicación y fechas ${ }^{3}$.

\section{COBib-Públicas: el sitio web}

El sitio web creado para la herramienta COBib-Públicas está estructurada según dos grandes bloques bien diferenciados: a la izquierda de la pantalla se disponen sus diferentes secciones o categorías; y en el bloque principal se muestra el contenido de las mismas. Todo ello se percibe mejor con una captura de su portada (figura 1).

Las secciones del menú lateral izquierdo incluyen:

- Inicio;

- las correspondientes para los siete períodos de tiempo en que se han distribuido las noticias;

- anexos;

- créditos.

Su contenido se explica a continuación.

\subsection{Inicio}

Se trata de la portada de la web. Incluye su título y un breve resumen. Puede verse en la captura de la figura 1.

\subsection{Secciones para los períodos por fecha}

Cada una de las siguientes siete secciones consisten en la clasificación de las noticias digitales recuperadas por períodos de tiempo. Los dos primeros períodos cubren en torno a un mes cada uno, mientras que los restantes, cubren en torno a 15 días. Así, los períodos delimitados que configuran las siete categorías son los siguientes: 


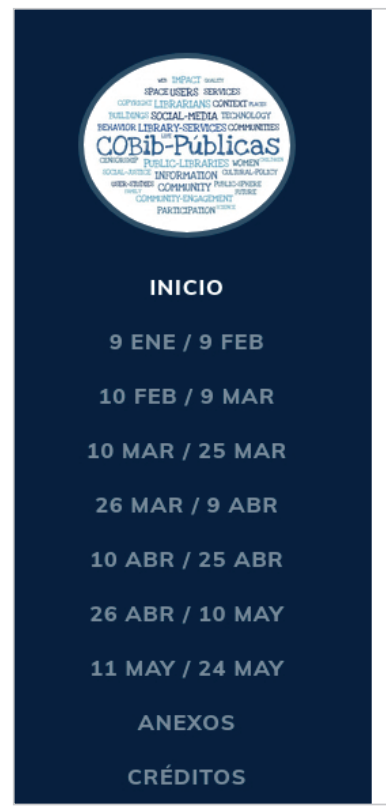

\title{
COBIB-PÚBLICAS: OBSERVATORIO DE LAS BIBLIOTECAS PÚBLICAS EN ESPAÑA DURANTE LA PANDEMIA POR COVID-19 BASADO EN NOTICIAS DIGITALES
}

Pedro Lázaro Rodriguez | pedrolr(at)ugr(dot)es | Secaba-Lab | Universidad de Granada

\begin{abstract}
En esta página web se presenta el proyecto COBib-Públicas. El observatorio se basa en 760 noticias publicadas en medios digitales de España sobre bibliotecas públicas desde inicios de enero hasta finales de mayo de 2020. El objetivo es que sirva como punto al que acudir para obtener información sobre las bibliotecas públicas durante la pandemia por Covid-19.
\end{abstract}

A la izquierda se encuentran las secciones para: el Inicio (o portada); los 7 períodos de tiempo en los que se ha dividido la difusión de noticias: los Anexos; y los Créditos. El proyecto se presentará vía ThinkEPI en forma de mensaje en la lista de distribución IWETEL. En la sección de Anexos pueden consultarse diversos documentos que incluyen la presentación de COBib-Públicas, con conjuntos de datos, una mirada general desde el observatorio, y otra personal.

Dado el carácter abierto y dinámico de la web, se podrán ir efectuando actualizaciones de aquí en adelante.|

Figura 1. Estructura de COBib-Públicas a través de su portada

- del 9 de enero al 9 de febrero;

- del 10 de febrero al 9 de marzo;

- del 10 al 25 de marzo;

- del 26 de marzo al 9 de abril;

- del 10 al 25 de abril;

- del 26 de abril al 10 de mayo;

del 11 al 24 de mayo.

Cada una de estas siete secciones incluye su título junto con el total de noticias en cada período, y una tabla dinámica en la que se muestran las noticias digitales recuperadas. Esta tabla incluye columnas para el título de cada noticia en hiperenlace con destino a la noticia, el medio digital en que fue publicada, y su fecha. Esta información es la dada por la base de datos MyNews y se ha respetado tal y como la ofrece dicha base de datos. Todo ello se puede ver mejor a partir de la figura 2 como ejemplo con la sección del primer período considerado.

Las tablas aparecen ordenadas por la columna de fecha para poder seguir su evolución, aunque pueden ordenarse también según las demás. La ordenación por la columna del título de las noticias

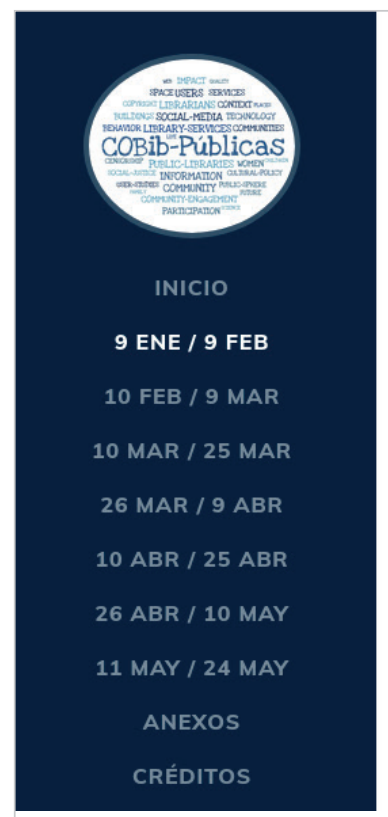

\section{ENERO / 9 FEBRERO (157 NOTICIAS)}

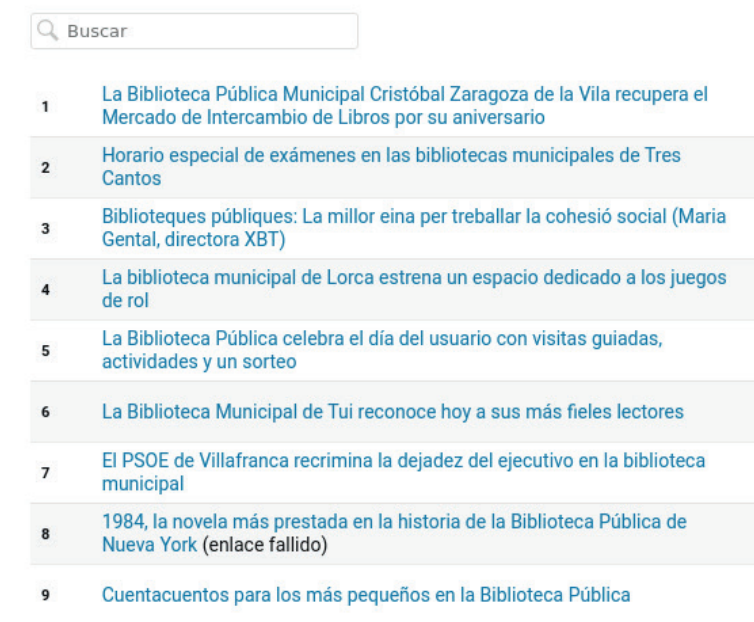

Figura 2. Contenido de las secciones de los períodos en que se clasifican las noticias 
establece la ordenación según el url del hiperenlace, y no según el texto del título de las noticias. En ese sentido, no se recomienda usar la ordenación por esta columna. En cambio, la posibilidad de ordenación resulta útil también en la columna de medios que publicaron las noticias, pues permite ver el listado de noticias por medio también detectando su volumen de producción.

Las tablas de las noticias incluyen un cuadro de búsqueda justo encima de la fila dedicada al encabezamiento de las columnas. Esta búsqueda permite filtrar las filas según la cadena de texto deseada. Por último, cabe señalar que de cada tabla se muestran las primeras 20 noticias. Se hace así para no saturar el sitio web y agilizar su lectura de una forma más amigable. Este número puede ampliarse a todas las de cada período pulsando el botón situado al pie de las tablas.

\subsection{Anexos}

Esta sección está relacionada con el contenido de este trabajo. Incluye información de las secciones sobre la lectura general y la lectura personal de los resultados y noticias incluidas en COBib-Públicas. Los contenidos serán añadidos en texto o en enlace a documentos, según se vaya desarrollando el observatorio en futuras actualizaciones.

\subsection{Créditos}

Se añaden referencias a los recursos empleados para la construcción de COBib-Públicas. Para su implementación web se ha utilizado un tema de Start Bootstrap (Start Bootstrap, 2020), con licencia del Instituto Tecnológico de Massachusetts (MIT). Por otro lado, las tablas con las noticias recuperadas se han diseñado con Datawrapper (Datawrapper, 2020). Por último, en esta sección de Créditos se incluye información del creador y licencias de la aplicación.

\section{Una mirada general desde el observatorio}

La búsqueda planteada en la sección de materiales devolvió un total de 761 noticias. El conjunto de noticias se analizó en busca de duplicados, hallándose solo uno en una noticia del medio europapress. es. Este duplicado se debe a que la noticia figura como publicada en los medios europapress.es y europapress.es/epsocial.aspx. Dado que ambas compartían url y título, se optó por eliminar el duplicado. Aparte de dicho caso, se detectaron tres pares de noticias que comparten url y título, pero que aparecen como publicadas cada par en medios diferentes. Por esa razón se optó por mantenerlas. También, se comprobaron los urls de todas las noticias detectándose nueve enlaces fallidos. No obstante, dado que figuran con título, fecha y medio de publicación, se optó por mantenerlas añadiendo entre paréntesis el fallo del enlace. En definitiva, el total de noticias válidas quedó en 760 . Su evolución por los períodos de tiempo definidos se puede ver en el gráfico 1.

Lo primero que hay que tener presente del gráfico es que los dos primeros períodos cubren en torno a un mes cada uno, mientras que los restantes cubren 15 días. La razón de haber diseñado así el gráfico y la distribución de períodos se debe a que las primeras noticias detectadas sobre bibliotecas públicas

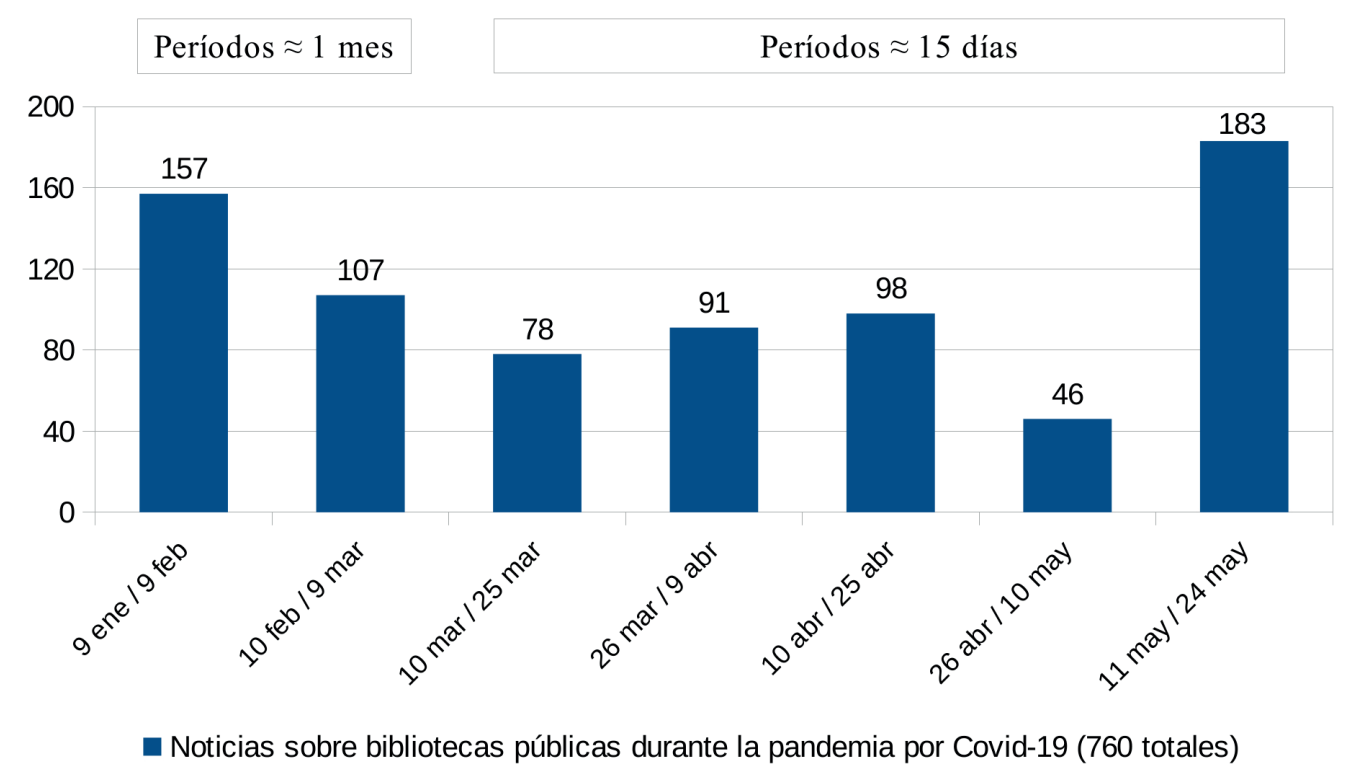

Gráfico 1. Evolución de las noticias digitales por período 
y Covid-19 son del 10 y 11 de marzo. El día 10 de marzo se detecta una en el medio lanzadigital.com con el título "Las Bibliotecas Municipales de Ciudad Real notan una leve reducción de visitas por el coronavirus" (Chinchilla, 2020). Para el día 11 de marzo se detectan ya hasta 5 noticias en medios diferentes sobre el cierre de bibliotecas en Madrid debido a la Covid-19. De esta forma, los períodos sin noticias sobre Covid-19 antes de esa fecha se dividen en dos con una duración de un mes cada uno, mientras que de ahí en adelante se han distribuido en cinco en torno a 15 días cada uno de ellos.

Desde el período que se inicia el 10 de marzo y hasta el 25 de abril, la publicación de noticias está entre 78 y 98 , cayendo a 46 entre el 26 de abril y el 10 de mayo. Lo que sí se ve claramente es que, en el último período, desde el 11 al 24 de mayo, la publicación de noticias crece hasta las 183. Como se puede ver en la sección para dicho período de COBib-Públicas, muchas de estas noticias tratan sobre la reapertura de bibliotecas.

En cuanto a los medios con más noticias, en la tabla 1 se muestran los 16 medios con más noticias publicadas.

El medio con más noticias publicadas es la agencia de noticias europapress.es, con 89 , seguida de elperiodic.com con 60 y de gentedigital.es con 50. La suma de noticias de los 16 medios anteriores es de 437 noticias, que representan en torno al $60 \%$ de todas las consideradas en COBib-Públicas.

Atendiendo a los nombres de los medios, se puede ver que la difusión de noticias sobre bibliotecas públicas ha sido más intensa en lugares como Cataluña (elperiodic.com y gencat.cat), Castilla y León (diariodesalamanca.es y salamanca24horas.com), o el País Vasco (diariovasco.com), Galicia (lavozdegalicia.es), Canarias (eldia.com), Extremadura (extremaduradehoy.com) o Castilla-La Mancha (lanzadigital. com). Todo ello se complementa también con medios de ámbito nacional, como por ejemplo abc.es, eldiario.es, cadenaser.es, o 20 minutos.es.

Los resultados de las noticias por medio podrían ponerse en relación con la realidad de las bibliotecas públicas por comunidades autónomas en cuanto a su uso y su presupuesto. No obstante, hay que tener presente que COBib-Públicas incluye noticias sobre bibliotecas públicas en medios digitales durante el período concreto de crisis sanitaria por la Covid-19. Para extraer conclusiones generales sobre la relación entre volumen de noticias de medios por ámbito geográfico y uso y presupuesto de bibliotecas, habría que considerar también medios impresos y la naturaleza de los medios digitales mismos. Estos medios digitales pueden ser consultados desde cualquier lugar con internet con mayor accesibilidad y facilidad que un medio impreso.

En Lázaro-Rodríguez, López-Gijón y Herrera-Viedma (2018) se planteaba una correlación entre las noticias publicadas sobre bibliotecas públicas en medios por comunidades autónomas y los préstamos y visitas a las mismas. La correlación no resultaba significativa, pero los autores mostraban cómo en algunas de las comunidades autónomas con más (y menos) préstamos y visitas, se estaban publicando más (y menos) noticias sobre bibliotecas públicas en los medios por ámbito geográfico.

A partir de COBib-Públicas, se puede proponer atender a la relación entre los resultados de los medios digitales con más publicaciones de la tabla 1 con la información sobre presupuesto y préstamos per cápita de las bibliotecas públicas españolas incluida en la sección Indicadores de la herramienta Secaba-Rank (Lázaro-Rodríguez et al., 2018). En ese sentido, hay que tener en cuenta que las noticias y medios de COBib-Públicas se refieren a 2020, y los datos más actuales de Secaba-Rank son de 2017 (Secaba-Rank, 2020). Estos datos también son los más actuales en la web de Bibliotecas públicas españolas en cifras del Ministerio de Cultura y Deporte $(M C D)$ de España $(M C D, 2020)$, fuente de datos que utiliza Secaba-Rank. Debido a la diferencia de años entre las dos realidades puestas en relación, se trata tan solo de una propuesta de estudio.

Cataluña es la comunidad autónoma donde más préstamos per cápita se producen según Secaba-Rank, y está también representada con dos medios en la tabla 1 sobre medios digitales con más noticias sobre bibliotecas públicas y Covid-19. Lo mismo sucede con Castilla y León, segunda tras Cataluña en préstamos per cápita en la herramienta Secaba-Rank, y que también aparece en la tabla 1 con dos medios digitales de Salamanca. País Vasco es la cuarta en préstamos per cápita, y aparece en la tabla 1 con un medio digital. Todo ello también se complementa con que Cataluña, País Vasco y 
Castilla y León son tres de las cuatro comunidades autónomas que más invierten en presupuesto para bibliotecas públicas de acuerdo con Secaba-Rank. La otra comunidad autónoma con mayor inversión es Castilla-La Mancha, que también aparece en la tabla 1 con un medio digital.

No obstante, también se puede atender al caso de Canarias, que según Secaba-Rank tiene los préstamos per cápita y el presupuesto más bajos de España, y que aparece en la tabla 1 con un medio. Este hecho puede plantearse como acción en aras de la mejora de las bibliotecas públicas en dicha comunidad autónoma.

A partir de todo lo anterior se puede plantear que en los medios digitales de las comunidades autónomas donde más se usan las bibliotecas (uso en cuanto a préstamos) y más se invierte (datos de 2017), se han difundido también más noticias sobre bibliotecas públicas durante la pandemia por Covid-19. Todo ello con el matiz en cuanto al caso de Canarias, que puede plantearse como caso de buenas prácticas por su esfuerzo de mejora para otras comunidades con préstamos y presupuesto bajos como puede ser Andalucía. Probablemente, los casos de comunidades autónomas con volumen alto de noticias publicadas y préstamos (y presupuesto) bajos, son los que rompían la correlación significativa entre las variables en el trabajo de Lázaro-Rodríguez, López-Gijón y Herrera-Viedma (2018).

La propuesta planteada aporta base a la idea que los autores anteriores también plantean acerca de que la visibilidad de las bibliotecas en medios puede concebirse como forma para aumentar su uso y los beneficios que generan a la sociedad. Pero siempre con la cautela de no considerar la relación lineal entre variables como causalidad. En ese sentido, hay que recordar también que se están considerando noticias sobre bibliotecas públicas en medios digitales durante la pandemia por Covid-19 y el uso de bibliotecas solo en relación con los préstamos y no con otras dimensiones como las visitas, actividades culturales, etc. Además, para alcanzar conclusiones más sólidas respecto a lo anterior, habrían de considerarse noticias en otros tipos de medios como los impresos y no solo durante la crisis sanitaria analizada.

\section{Una mirada personal desde el observatorio}

La mirada personal planteada en esta sección complementa a la general anterior. Se trata de un recorrido por las noticias clasificadas según los períodos considerados y atendiendo a su contenido. Por su carácter más subjetivo resaltando lo que más ha llamado la atención, se plantea como personal.

Tal y como se ha dicho en la sección anterior, las primeras noticias detectadas sobre bibliotecas públicas en relación con todo lo generado por la Covid-19 son del 10 y 11 de marzo. Se refieren a la reducción de las visitas en Ciudad Real y a la suspensión de la apertura al público de la red de bibliotecas de la Comunidad de Madrid. El 10 de marzo es justo el día de inicio del tercer período que se definió en COBib-Públicas. Esto significa que en los dos períodos anteriores que van desde el 9 de enero hasta el 9 de marzo, no se detecta noticia alguna en relación con la Covid-19 y bibliotecas públicas. Lo anterior no quiere decir que en las bibliotecas no se llevaran a cabo iniciativas informativas respecto a la crisis sanitaria que se avecinaba, pues puede ser que no se plantearan en forma de noticia en medios digitales. No obstante, sí que puede pensarse que, si se hubiesen planteado muchas iniciativas, muy probablemente se hubiesen visto reflejadas en forma de noticias. La realidad es que la pandemia por Covid-19 y sus efectos sobrevinieron con celeridad y sin preparación y acción en muchos países y sectores, por no decir todos, y en las bibliotecas españolas puede reconocerse que sucedió lo mismo.

En los tres períodos que van desde el 10 de marzo hasta el 25 de abril, el tema predominante es el cierre físico de bibliotecas y la adaptación a los servicios digitales. Estos temas encajan con el Decreto del estado de alarma del 14 de marzo y el confinamiento en España. La temática de las noticias en dichos períodos gira también en torno a:

- el aumento de presupuesto;

- adquisiciones de licencias de libros electrónicos, el aumento de su uso y la gratuidad de servicios relacionados con ello;

- el registro de nuevas personas usuarias;

- iniciativas de bibliotecas en redes sociales o plataformas como Youtube.

También, destacan noticias referidas a:

- 23 de abril por el día del libro;

- sobre impresoras 3D para fabricar material ante la Covid-19;

- sobre la adaptación de edificios de bibliotecas para comisarías o con fines sanitarios. 
Con todo, el gran protagonismo de lo digital puede servir para analizar en estudios futuros si las bibliotecas españolas están ofreciendo servicios que cubren las expectativas de la población al respecto.

Pasando al período que va desde el 26 de abril al 10 de mayo, se detectan las primeras noticias sobre la reapertura de bibliotecas. Por ejemplo, el 30 de abril se publica una noticia en elperiodic.com con el título "El archivo y las bibliotecas municipales de Alcoy abrirán progresivamente a partir del lunes 4 de mayo" (elperiodic.com, 2020). En un sentido similar, el 4 de mayo se publica una noticia en elperiodicoextremadura.com con el título "La biblioteca municipal de Plasencia abrirá el día 11 con el aforo reducido al 30\%" (Rodríguez-Muñoz, 2020). Esta dinámica de noticias sobre la reapertura de las bibliotecas se da también en el último período considerado (11 a 24 de mayo).

La temática por períodos planteada en los párrafos anteriores encaja con el transcurrir y el desarrollo de la crisis sanitaria y las medidas que se han tenido que tomar en España en cuanto al confinamiento y las fases de la llamada desescalada. Pero también se pueden destacar otros temas planteados en las noticias. Por ejemplo, el 3 de abril se publican dos noticias en elpais.com y lavanguardia.es con los títulos respectivos "Elogio de la biblioteca pública" (Vicente, 2020) y "Mi patria ha sido la biblioteca municipal de Barberà del Vallès" (Amela, 2020). También, el 11 de abril se publica en elmundo.es la noticia con título "Frederick Wiseman: Una biblioteca pública es también una vacuna contra la pandemia" (elmundo.es, 2020). Por último, eldiario.es publica una el 11 de mayo con el título "El silencio de las bibliotecas públicas", que consiste en una reflexión sobre su función social (López-Higueras-Escobar, 2020). Estas noticias sirven para ejemplificar el valor de las bibliotecas públicas, reconocido en este caso en tiempos de crisis sanitaria, pero que ha de extrapolarse a cualquier otro tiempo y lugar. En ese sentido, siguiendo la idea de la noticia en elmundo.es que plantea a las bibliotecas públicas como antídoto o vacuna contra la pandemia (elmundo.es, 2020), puede trabajarse para la consolidación de las bibliotecas como vacuna para un mundo mejor más allá de una crisis sanitaria concreta.

Por último, otro tema a resaltar detectado desde mediados de abril es el de la construcción de nuevas bibliotecas. Por ejemplo, el 23 de abril se publica en diariodeavila.es la noticia con título "La Adrada estenará [sic] Biblioteca Pública tras el Covid-19" (diariodeavila.es, 2020). En un sentido similar, el 28 de abril se publica en diariosur.es la noticia con título "Nerja activa los trámites para construir una nueva biblioteca pública" (Cabezas, 2020). Estas noticias pueden ser también signo de reconocimiento del valor de las bibliotecas públicas planteado en el párrafo anterior.

\section{Consideraciones finales}

En este trabajo se ha presentado COBib-Públicas como observatorio de las bibliotecas públicas en España durante la pandemia por Covid-19 basado en noticias digitales. El observatorio se concibe como punto al que acudir para obtener información al respecto. Se ha propuesto una mirada general desde el observatorio, y otra personal, pero la idea es que cada persona interesada pueda realizar también su propia mirada o lectura gracias a la clasificación de las noticias propuesta.

La mirada general desde el observatorio incluye información sobre la evolución del volumen de noticias publicadas por período y sobre los medios que más publican. Este segundo punto incluye una propuesta de análisis en relación con los préstamos y presupuesto para bibliotecas públicas por comunidades autónomas. No obstante, para poder alcanzar conclusiones más completas y sólidas se habrían de considerar noticias en otro tipo de medio como los impresos y no solo sobre la Covid-19. Por su lado, la mirada personal se ha centrado en comentar diversas temáticas detectadas en las noticias con base en un recorrido temporal por las mismas.

COBib-Públicas se ha implementado en la web y por su carácter abierto y dinámico puede ir actualizándose de aquí en adelante. En la sección de Anexos podrán añadirse documentos de trabajo, incluyendo nuevos análisis e incluyendo períodos con nuevas noticias sobre bibliotecas públicas que se publiquen de aquí en adelante. Con todo, por su contenido y forma, COBib-Públicas puede resultar interesante para personas dedicadas a la investigación en biblioteconomía y documentación, por ejemplo, como base para futuras investigaciones; para las profesionales de las bibliotecas y para la población en general.

\section{Notas}

1. Acceso desde la biblioteca electrónica de la Universidad de Granada.

2. Se puede acceder al proyecto desde: https://osf.io/6dtjv

3. El archivo csv se encuentra disponible en el siguiente url dentro del proyecto: https://osf.io/hzwx4 


\section{Referencias}

Amela, Víctor M. (2020). "Mi patria ha sido la biblioteca municipal de Barberà del Vallès". La vanguardia, 3 abril. https://www.lavanguardia.com/lacontra/20200403/48278949045/mi-patria-ha-sido-la-biblioteca-municipal-debarbera-del-valles.html

Andreu-Sánchez, Celia; Martín-Pascual, Miguel-Ángel (2020). "Fake images of the SARS-CoV-2 coronavirus in the communication of information at the beginning of the first Covid-19 pandemic". El profesional de la información, v. 29, n. 3, e290309.

https://doi.org/10.3145/epi.2020.may.09

Angulo-Marcial, Noel (2009). “¿Qué son los observatorios y cuáles son sus funciones?". Innovación educativa, v. 9, n. 47, pp. 5-17.

https://www.redalyc.org/articulo.oa?id=179414895002

Cabezas, Eugenio (2020). "Nerja activa los trámites para construir una nueva biblioteca pública". Diario sur, 28 abril.

https://www.diariosur.es/axarquia/nerja-activa-tramites-20200428143737-nt.html

Casero-Ripollés, Andreu (2020). "Impact of Covid-19 on the media system. Communicative and democratic consequences of news consumption during the outbreak". El profesional de la información, v. 29, n. 2, e290223. https://doi.org/10.3145/epi.2020.mar.23

Chinchilla, Juan-Carlos (2020). “Las Bibliotecas Municipales de Ciudad Real notan una leve reducción de visitas por el coronavirus". lanzadigital.com, 10 marzo.

https://www.lanzadigital.com/provincia/ciudad-real/las-bibliotecas-municipales-notan-una-leve-reduccion-devisitas-por-el-coronavirus

Costa-Sánchez, Carmen; López-García, Xosé (2020). “Comunicación y crisis del coronavirus en España. Primeras lecciones". El profesional de la información, v. 29, n. 3, e290304.

https://doi.org/10.3145/epi.2020.may.04

Datawrapper (2020). Create charts and maps with Datawrapper.

https://www.datawrapper.de

diariodeavila.es (2020). "La Adrada estenará (sic) Biblioteca Pública tras el Covid-19". Diario de Ávila, 23 abril.

https://www.diariodeavila.es/Noticia/Z577B304A-BFAF-5025-AA909ACA2D6D4939/202004/La-Adrada-estenaraBiblioteca-Publica-tras-el-Covid-19

elmundo.es (2020). "Frederick Wiseman: 'Una biblioteca pública es también una vacuna contra la pandemia'". elmundo.es, 11 abril.

https://www.elmundo.es/cultura/cine/2020/04/11/5e8c53f3fc6c83b75e8b46bb.html

elperiodic.com (2020). “El archivo y las bibliotecas municipales de Alcoy abrirán progresivamente a partir del lunes 4 de mayo". elperiodic.com, 30 abril.

https://www.elperiodic.com/alcoilarchivo-bibliotecas-municipales-alcoy-abriran-progresivamente-partir-lunesmayo_678149

Lázaro-Rodríguez, Pedro; Herrera-Viedma, Enrique (2020). "Noticias sobre Covid-19 y 2019-nCoV en medios de comunicación de España: el papel de los medios digitales en tiempos de confinamiento". El profesional de la información, v. 29, n. 3, e290302.

https://doi.org/10.3145/epi.2020.may.02

Lázaro-Rodríguez, Pedro; López-Gijón, Javier; Alonso, Sergio; Martínez-Sánchez, María-Ángeles; Herrera-Viedma, Enrique (2018). "Secaba-Rank, herramienta online para analizar y evaluar bibliotecas". El profesional de la información, v. 27, n. 2, pp. 278-288. https://doi.org/10.3145/epi.2018.mar.06

Lázaro-Rodríguez, Pedro; López-Gijón, Javier; Herrera-Viedma, Enrique (2018). “Visibilidad de las bibliotecas públicas y la lectura en medios de comunicación españoles frente a otros hechos de la cultura y relación con su uso: medidas para su mayor promoción y difusión". BiD: textos universitaris de biblioteconomia i documentació, n. 40. https://doi.org/10.1344/BiD2018.40.7

López-Higueras-Escobar, David (2020). "El silencio de las bibliotecas públicas". eldiario.es, 11 mayo. https://www.eldiario.es/opinionsocios/silencio-bibliotecas-publicas_6_1026157415.html

Masip, Pere; Aran-Ramspott, Sue; Ruiz-Caballero, Carlos; Suau, Jaume; Almenar, Ester; Puertas-Graell, David (2020). "Consumo informativo y cobertura mediática durante el confinamiento por el Covid-19: sobreinformación, sesgo ideológico y sensacionalismo". El profesional de la información, v. 29, n. 3, e290312. https://doi.org/10.3145/epi.2020.may.12

MCD (2020). Bibliotecas públicas españolas en cifras. Ministerio de Cultura y Deporte. http://www.culturaydeporte.gob.es/cultura/areas/bibliotecas/mclebp/portada.html 
Pérez-Dasilva, Jesus-Angel; Meso-Ayerdi, Koldobika; Mendiguren-Galdospín, Terese (2020). "Fake news y coronavirus: detección de los principales actores y tendencias a través del análisis de las conversaciones en Twitter". El profesional de la información, v. 29, n. 3, e290308.

https://doi.org/10.3145/epi.2020.may.08

Rodríguez-Muñoz, Raquel (2020). “La biblioteca municipal de Plasencia abrirá el día 11 con el aforo reducido al $30 \% "$. El periódico. Extremadura, 4 mayo.

https://www.elperiodicoextremadura.com/noticias/plasencia/biblioteca-municipal-plasencia-abrira-dia-11-afororeducido-30_1231604.html

Salaverría, Ramón; Buslón, Nataly; López-Pan, Fernando; León, Bienvenido; López-Goñi, Ignacio; Erviti, María-Carmen (2020). "Desinformación en tiempos de pandemia: tipología de los bulos sobre la Covid-19". El profesional de la información, v. 29, n. 3, e290315.

https://doi.org/10.3145/epi.2020.may.15

Secaba-Rank (2020). "Indicadores". SECABA-Rank Públicas. Ranking de las redes de lectura pública españolas. http://secaba.ugr.es/rank/publicas/index.php/indicadores

Start Bootstrap (2020). Free bootstrap themes, templates, snippets, and guides. https://startbootstrap.com/

Vicente, Álex (2020). “Elogio de la biblioteca pública”. elpais.com, 3 abril. https://elpais.com/cultura/2020/04/03/babelia/1585933054_680060.htm/

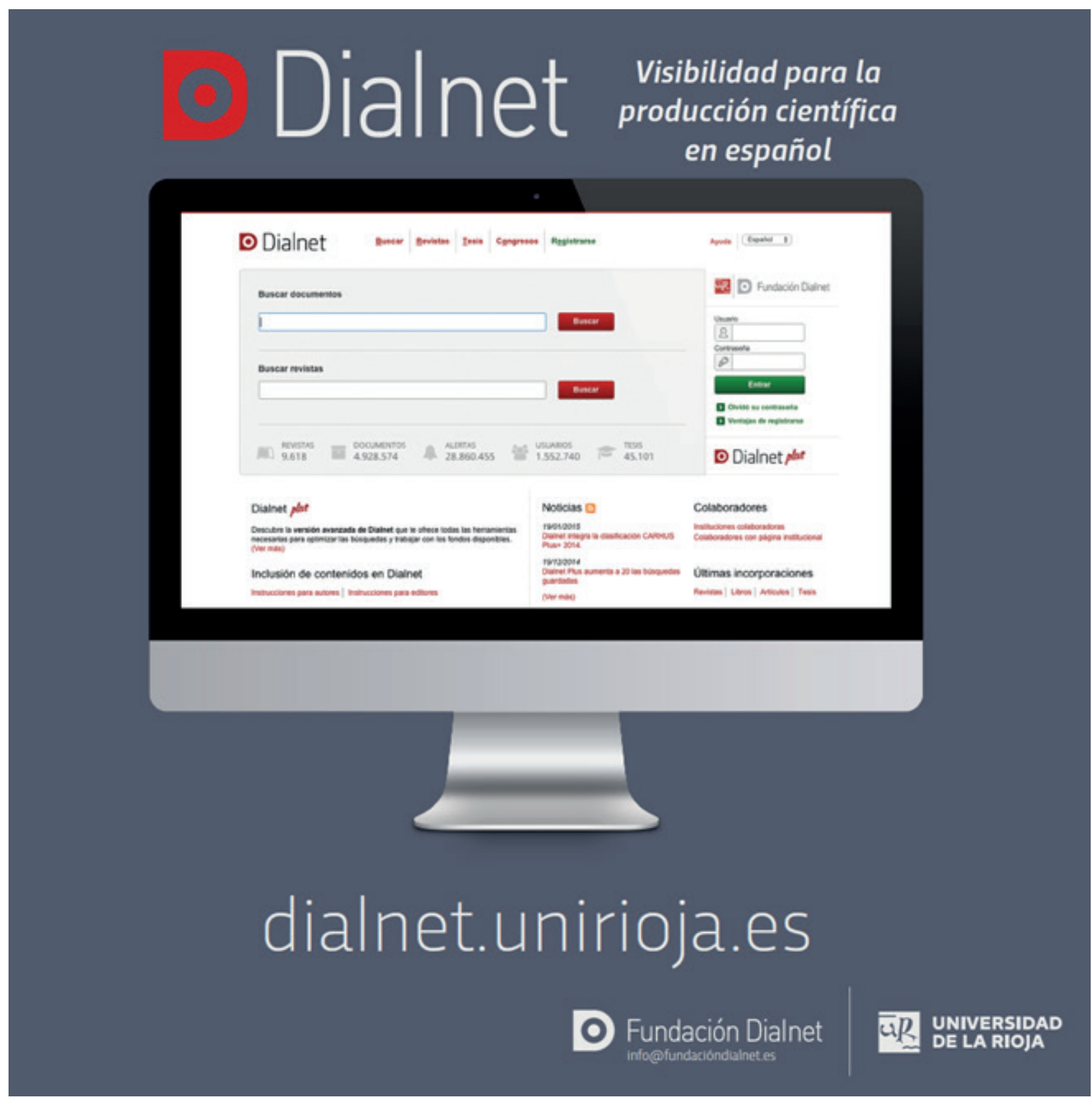


